\title{
Affinity of extracellular phosphatases for ELF97 phosphate in aquatic environments
}

\author{
Jiři Nedoma ${ }^{\mathrm{A}, \mathrm{D}}$, France Van Wambeke ${ }^{\mathrm{B}}$, Alena Štrojsováa, ${ }^{\mathrm{A}, \mathrm{C}}$ \\ Martina Štrojsová ${ }^{\mathrm{A}, \mathrm{C}}$ and Solange Duhamel ${ }^{\mathrm{B}}$ \\ A Biological Centre of the Academy of Sciences of the Czech Republic, v.v.i., Hydrobiological Institute, \\ Na sádkách 7, 37005 České Budějovice, Czech Republic. \\ B Laboratoire de Microbiologie Géochimie et Ecologie Marines, CNRS-UMR 6117, Campus de Luminy, \\ case 901, 13288 Marseille cedex 9, France. \\ CFaculty of Biological Sciences, University of South Bohemia, Branišovská 31, \\ 37005 České Budějovice, Czech Republic. \\ DCorresponding author. Email: nedoma@hbu.cas.cz
}

\begin{abstract}
Recently, the phosphatase substrate ELF97 phosphate (ELFP) has been employed to study the presence of extracellular phosphatases in different plankton populations in natural aquatic environments. Kinetic properties of ELFP hydrolysis by natural extracellular phosphatases are, however, mostly unknown. We indirectly studied the affinity of extracellular phosphatases for ELFP in different aquatic environments through its ability to inhibit the hydrolysis of 4-methylumbelliferyl phosphate (4MUP). Values of inhibition constants, $K_{\mathrm{i}}$, which correspond to the concentrations necessary for half saturation of phosphatases by ELFP, were lowest $\left(0.18-4.5 \mu \mathrm{mol} \mathrm{L}^{-1}\right)$ in the oligotrophic Mediterranean Sea. We found higher values (i.e. lower affinity) in oligo- to mesotrophic acidified lakes $\left(5.2-14 \mu \mathrm{mol} \mathrm{L}^{-1}\right)$, in a eutrophic reservoir $\left(13-35 \mu \mathrm{mol} \mathrm{L}^{-1}\right)$ and in a pure culture of the marine bacterium Alteromonas infernus $\left(29 \mu \mathrm{mol} \mathrm{L}^{-1}\right)$. ELFP had a pronounced effect on the parameter $K_{\mathrm{M}}$ (Michaelis constant) of 4MUP saturation kinetics, while its effect on the parameter $V_{\max }$ was low. This behaviour is compatible with the assumption of competitive interaction between 4MUP and ELFP. Our experiments indicated that the assay ELFP concentration in the detection kit used was $250-500 \mu \mathrm{mol} \mathrm{L}^{-1}$ (after the recommended dilution to a ratio of 1:20), which would ensure $>99 \%$ saturation of extracellular phosphatases in marine environments and $>90 \%$ saturation in the studied fresh waters.
\end{abstract}

Additional keywords: freshwater, marine.

\section{Introduction}

Phosphorus availability is considered to be a factor that limits productivity in many freshwater and certain marine systems (e.g. Schindler 1977; Elser et al. 1995; Van Wambeke et al. 2002). During periods of phosphorus limitation, concentrations of dissolved inorganic phosphorus, the only directly bioavailable phosphorus form, drop regularly to negligible values (Taylor and Lean 1991; Moutin et al. 2002). Synthesis of inducible extracellular phosphatases is a common adaptive response of microbial cells to phosphorus shortage (Gage and Gorham 1985; Jansson et al. 1988; Hoppe 2003); however, phosphorus stress might not be the only motive for their synthesis (Hoppe 2003) and non-microbial origin of phosphatases may be important in particular cases (Jean et al. 2003). Phosphatases cleave molecules of organic esters of phosphoric acid into orthophosphate and organic parts, thus converting the mostly non-bioavailable dissolved organic phosphorus to inorganic phosphorus. Recently, the phosphatase substrate ELF97 phosphate (ELFP) was employed to study the presence of extracellular phosphatases in different plankton populations in natural aquatic environments (González-Gil et al. 1998; Rengefors et al. 2001; Štrojsová et al. 2003, 2005).
The product of ELFP hydrolysis, a brightly fluorescent ELF97 alcohol (ELFA), is water-insoluble and precipitates at the site of its origin, thus labelling cells with phosphatase activity.

Very little is known about the kinetics of ELFP hydrolysis by extracellular phosphatases in natural aquatic systems. The ELFP hydrolysis is only an initial step of a complicated process leading finally (and not necessarily) to the formation of observable ELFA precipitates, and these two processes may be decoupled. Moreover, the quantification of the fluorescence of ELFA precipitates is hindered by the low ELFA fluorescence yield and by the complicated methodology. The affinity of extracellular phosphatases for ELFP is therefore not known and, as a consequence, neither is the degree of phosphatase saturation by ELFP in most experiments. Experiments with conventional substrates like 4MUP suggest a great variability in substrate affinity in different aquatic systems (e.g. differences in $K_{\mathrm{M}}$ of three orders of magnitude for 4MUP among lakes, Nedoma et al. 2003a).

Most frequently, ELFP is applied as the part of a special 'endogenous phosphatase detection kit', developed originally for use in histochemistry by Molecular Probes (Cox and Singer 1999). However, the concentration of ELFP used in the kit is 
Table 1. Basic parameters of the samples used in this study, and kinetic parameters ( \pm s.d.) $K_{M}$ and $V_{\max }$ for 4-methylubelliferyl phosphate (4MUP) hydrolysis and inhibition constants $K_{\mathrm{i}}$ for inhibition of 4MUP hydrolysis by ELF97 phosphate (ELFP)

4MUP range $=$ range of 4MUP concentrations used in saturation experiments; $K_{\mathrm{i}}=$ inhibition constant determined in displacement experiments using pure ELFP; NWM = north-west Mediterranean; b.d.l. = below the detection limit of $30 \mathrm{nmol} \mathrm{L}^{-1} ;$ n.a. $=$ not available

\begin{tabular}{|c|c|c|c|c|c|c|c|c|}
\hline Date & Locality (source) & $\begin{array}{c}\text { Temp } \\
{ }^{\circ} \mathrm{C}\end{array}$ & $\mathrm{pH}$ & $\begin{array}{c}\text { SRP } \\
\mathrm{nmol} \mathrm{L}^{-1}\end{array}$ & $\begin{array}{c}V_{\max } \\
\mathrm{nmol} \mathrm{L}^{-1} \mathrm{~h}^{-1}\end{array}$ & $\begin{array}{c}4 \mathrm{MUP} \\
K_{\mathrm{M}} \\
\mu \mathrm{mol} \mathrm{L}^{-1}\end{array}$ & $\begin{array}{l}\text { 4MUP range } \\
\mu \mathrm{mol} \mathrm{L}^{-1}\end{array}$ & $\begin{array}{c}\text { ELFP } \\
K_{\mathrm{i}} \\
\mu \mathrm{mol} \mathrm{L}^{-1}\end{array}$ \\
\hline 8 Oct 04 & DYFAMED station (NWM) & 21 & 8.3 & 35 & $2.6 \pm 0.1$ & $0.076 \pm 0.011$ & $0.01-2$ & $0.44 \pm 0.12$ \\
\hline 10 Oct 04 & DYFAMED station & 20 & 8.3 & 35 & $2.7 \pm 0.1$ & $0.069 \pm 0.009$ & $0.01-2$ & $0.55 \pm 0.22$ \\
\hline 15 Oct 04 & DYFAMED station & 19 & 8.3 & 35 & $2.7 \pm 0.1$ & $0.082 \pm 0.008$ & $0.01-2$ & $0.18 \pm 0.04$ \\
\hline 26 May 05 & SOFCOM station (Marseille Bay) & 17 & 8.3 & 30 & $31 \pm 2$ & $0.41 \pm 0.09$ & $0.2-40$ & $4.5 \pm 1.7$ \\
\hline 1 Feb 05 & Plešné Lake (Czech Republic) & 0 & 4.9 & b.d.l. & $1600 \pm 100$ & $1.3 \pm 0.4$ & $0.2-20$ & $5.2 \pm 0.4$ \\
\hline 22 Feb 05 & Čertovo Lake (Czech Republic) & 0 & 4.4 & 35 & $700 \pm 50$ & $2.9 \pm 0.7$ & $0.2-20$ & $14 \pm 2$ \\
\hline 14 Mar 05 & Čertovo Lake & 0 & 4.5 & 30 & $260 \pm 20$ & $1.4 \pm 0.6$ & $0.2-40$ & $12 \pm 5$ \\
\hline 18 May 05 & Čertovo Lake & 9.5 & 4.6 & b.d.l. & $270 \pm 20$ & $1.6 \pm 0.5$ & $0.2-40$ & $13 \pm 2$ \\
\hline 13 Sep 05 & Ř́mov Reservoir (Czech Republic) & 19 & 8.0 & 115 & $53 \pm 4$ & $1.2 \pm 0.3$ & $0.025-20$ & $16 \pm 5$ \\
\hline 22 Sep 05 & Římov Reservoir & 14.5 & 6.9 & 95 & $28 \pm 2$ & $1.2 \pm 0.3$ & $0.025-20$ & $23 \pm 10$ \\
\hline 4 Oct 05 & Římov Reservoir & 14 & 7.1 & 220 & $31 \pm 11$ & $5.7 \pm 5.1$ & $0.025-20$ & $35 \pm 4$ \\
\hline 26 May 05 & Bacterial culture Alteromonas infernus & 20 & 7.5 & n.a. & $417 \pm 5$ & $0.23 \pm 0.02$ & 2-100 & $29 \pm 5$ \\
\hline
\end{tabular}

not known, which limits the interpretation of results. Pure ELFP of a known concentration was successfully employed in some studies as an alternative to the kit ELFP (Nedoma et al. 2003b; Štrojsová et al. 2003, 2005).

One possible way to obtain information about the kinetics of ELFP hydrolysis by naturally occurring extracellular phosphatases is to study the kinetics indirectly, studying the interaction of ELFP with the hydrolysis of another substrate that is readily measurable, e.g. 4MUP, and by employing the theoretical background developed for studying enzyme inhibition (Dixon and Webb 1964). Within the framework of this approach, 4MUP acts as a substrate and ELFP as an inhibitor. Analysis of the inhibition kinetics enables the estimation of the inhibition constant, $K_{\mathrm{i}}$, which is a measure of enzyme affinity for the inhibitor. Thus, we may indirectly estimate phosphatase affinity for ELFP, as well as the concentration of ELFP in the endogenous phosphatase detection kit, by comparing its efficiency in the inhibition of 4MUP hydrolysis with the efficiency of pure ELFP of known concentration used as a standard. Another intriguing question, not yet answered (or raised), is whether the enzymes that hydrolyse ELFP and 4MUP (as well as other conventional substrates) in natural environments are identical. Implicitly, a positive answer is assumed but there is little experimental evidence on the subject (Štrojsová et al. 2003).

The specific aims of the experiments described in the present study were to indirectly determine through inhibition of 4MUP hydrolysis (1) the affinity of extracellular phosphatases in different aquatic environments towards ELFP, and (2) whether the same enzymes hydrolyse ELFP and 4MUP.

\section{Materials and methods}

\section{Samples}

We used water samples from different marine and freshwater environments taken from 5-m (marine) and $0.5-\mathrm{m}$ depth (freshwater). Additionally, a pure bacterial culture was also examined. Some relevant characteristics of samples are given in Table 1. The Dynamique des Flux de Matire en Mediterrane (DYFAMED) station is situated in the Western Mediterranean Sea, $\sim 52$ kilometres south of Nice $\left(43^{\circ} 25^{\prime} \mathrm{N}, 07^{\circ} 52^{\prime} \mathrm{E}\right.$; depth $2350 \mathrm{~m})$. A nitrate-to-phosphate ratio of $\sim 20$ in deep waters and thermal stratification in summer and autumn creates a situation of strong phosphorus limitation (Marty et al. 2002). The Service d'Observation du Centre d'Ocanologie (SOFCOM) station is located in the southern part of Bay of Marseille, France $\left(43^{\circ} 18^{\prime} \mathrm{N}, 5^{\circ} 24^{\prime} \mathrm{E}\right.$; depth $\left.30 \mathrm{~m}\right)$ and it is under greater anthropogenic influence.

The Lakes Čertovo and Plešné are small, acidified mountain lakes (maximum depths 34 and $17 \mathrm{~m}$ respectively) located in the Bohemian Forest, Czech Republic $\left(30^{\circ} 12^{\prime} \mathrm{E}, 49^{\circ} 10^{\prime} \mathrm{N}\right)$ at altitudes of $1040-1080 \mathrm{~m}$. Lakes are permanently phosphorusdepleted owing to aluminium-phosphorus co-precipitation, which removes it from the water column (Kopáček et al. 2000). The eutrophic Rímov reservoir is located on the river Malše in South Bohemia $\left(49^{\circ} 10^{\prime} \mathrm{N}, 15^{\circ} 46^{\prime} \mathrm{E}\right.$; maximum depth $43 \mathrm{~m}$, altitude $470 \mathrm{~m}$ ), Czech Republic. Phosphorus limitation develops regularly in the epilimnion in spring and summer (Vrba et al. 1993, 1995; Štrojsová et al. 2003).

The marine bacterial strain Alteromonas infernus $(\gamma-$ proteobacteria), possessing inducible extracellular phosphatases, was cultivated in batch conditions in an artificial seawater medium that contained pyruvate $\left(0.33 \mathrm{mmol} \mathrm{L}^{-1} \mathrm{C}\right)$ and orthophosphate $\left(0.825 \mu \mathrm{moL}^{-1} \mathrm{P}\right)$ as single carbon and phosphorus sources respectively. We conducted experiments during the stationary phase of culture growth after strong phosphorus limitation had developed.

\section{Chemicals}

We purchased ELF97 phosphate (ELFP) from the producer, Molecular Probes (Eugene, OR, USA), either as a pure substance $\left(5 \mathrm{mmol} \mathrm{L}^{-1}\right.$ water solution, $0.2-\mu \mathrm{m}$ filtered, Catalogue No. E-6588), abbreviated as ELFP PURE hereafter, or as a part of the endogenous phosphatase detection kit (Catalogue No. E6601, Component A, Phosphatase substrate, $20 \times$ concentrate, 
concentration not specified, in $2 \mathrm{mmol} \mathrm{L}^{-1}$ sodium azide), abbreviated hereafter as ELFP ${ }^{\mathrm{KIT}}$. We stored the original (supplied) solutions of ELFPPURE and ELFP ${ }^{\mathrm{KIT}}$ at $-20^{\circ} \mathrm{C}$ and $+4^{\circ} \mathrm{C}$, respectively, and filtered them through a spin filter of $0.2-\mu \mathrm{m}$ porosity just before use. The 4-methylumbelliferyl phosphate disodium salt (4MUP) and 4-methylumbelliferone (4MU) were purchased from Sigma (www.sigma.com).

\section{Extracellular phosphatase activity}

We fluorimetrically determined extracellular phosphatase activity using 4MUP as a substrate (Hoppe 1983) and 4MU as a standard. We performed incubations in duplicates, in the dark and at in situ temperature, adding $25 \mu \mathrm{L}$ of the substrate (4MUP) and 25 or $75 \mu \mathrm{L}$ of the inhibitor (ELFP diluted in $\mathrm{H}_{2} \mathrm{O}$, replaced by $\mathrm{H}_{2} \mathrm{O}$ in controls) to $2.5 \mathrm{~mL}$ of water sample. In parallel, we conducted calibrations relating fluorescence to $4 \mathrm{MU}$ concentration in each sample. Owing to specific requirements imposed by the character of samples ( $\mathrm{pH}$, enzyme activity) and the laboratory equipment available, the details of the method slightly differed for marine (Van Wambeke et al. 2002) and freshwater (Vrba et al. 1995) samples and for the bacterial culture. For marine samples, we measured fluorescence at in situ $\mathrm{pH}$ (8.3) for up to $6 \mathrm{~h}$. For freshwater samples, incubations lasted from 15 to $240 \mathrm{~min}$ at in situ $\mathrm{pH}$, and were completed by the addition of $100 \mu \mathrm{L}$ of $0.2 \mathrm{~mol} \mathrm{~L}^{-1} \mathrm{HgCl}$ (present from the beginning in blanks). We alkalised samples by the addition of $100 \mu \mathrm{L}$ of the mixture of $2 \mathrm{~mol} \mathrm{~L}^{-1} \mathrm{NaOH}+0.2 \mathrm{~mol} \mathrm{~L}^{-1}$ EDTA (final $\mathrm{pH}>11$ ) before fluorescence measurement. In bacterial cultures (buffered to $\mathrm{pH} 7.5$ with $10 \mathrm{mmol} \mathrm{L}^{-1} \mathrm{TRIS} / \mathrm{HCl}$ ), we read fluorescence throughout 2 -h incubations.

\section{Inhibition experiments}

We performed two types of inhibition experiments: 'displacement experiments' and 'saturation experiments'. We use these terms throughout the present study to distinguish two different experimental designs: (1) displacement experiments, in which we used different ELFP concentrations and a single 4MUP concentration (Fig. 1a), and (2) saturation experiments, in which we used a single ELFP concentration and different 4MUP concentrations (Fig. 1b). In each inhibition experiment, both ELFPPURE and ELFP ${ }^{\mathrm{KIT}}$ were applied in parallel at the same dilution (sets of dilutions) of the original solutions delivered by the manufacturer. The ELFP concentration referred to e.g. as $10^{-3}$ therefore means a final assay ELFP concentration that is 1000-times lower than that of the original solution delivered. In the case of ELFP PURE , supplied as $5 \mathrm{mmol} \mathrm{L}^{-1}$ solution, this corresponds to $5 \mu \mathrm{mol} \mathrm{L}^{-1}$. Consequently, the constants $K_{\mathrm{i}}^{\mathrm{PURE}}$ and $K_{\mathrm{i}}^{\mathrm{KIT}}$, characterising affinity of ELFP ${ }^{P U R E}$ and ELFP ${ }^{\mathrm{KIT}}$ to phosphatases, are given in units of ELFP dilution as well. In the case of $K_{\mathrm{i}}^{\mathrm{PURE}}$, the values can be recalculated to molar units as above.

\section{Displacement experiments}

In inhibition experiments of the displacement type, we examined the effects of ELFP, applied in a series of concentrations, on the hydrolysis velocity of a substrate (i.e. 4MUP) applied at a single low concentration (Fig. 1a). We kept 4MUP concentration, $S$, as low as possible, preferably $S<K_{\mathrm{M}}\left(K_{\mathrm{M}}\right.$ is the Michaelis constant for the 4MUP hydrolysis by extracellular phosphatases present
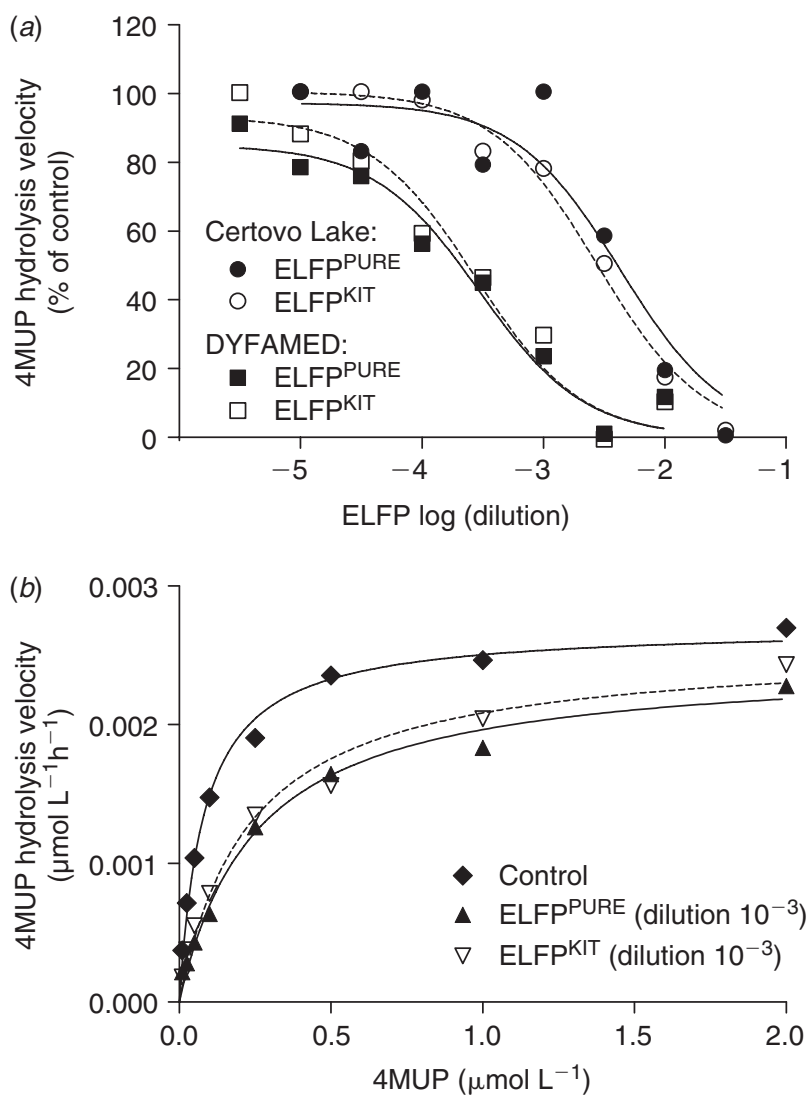

Fig. 1. (a) Examples of displacement curves: inhibition of hydrolysis of 4-methylumbelliferyl phosphate (4MUP) by ELF97 phosphate, applied at different concentrations, in samples taken at the DYFAMED station (northwest Mediterranean) on 10 October 2004, and in Lake Črtovo on 14 March 2005. Final concentration of 4MUP was $0.1 \mu \mathrm{mol} \mathrm{L}^{-1}$ in a marine sample and $1 \mu \mathrm{mol} \mathrm{L}^{-1}$ in a lake sample. ELFP concentrations are given as logarithm of dilution of the original solutions purchased from the manufacturer (Molecular Probes), either as a part of the endogenous phosphatase detection kit (ELFPKIT ; open symbols) or as a pure substance (ELFPPURE; closed symbols). (b) An example of a saturation experiment: a comparison of effects of ELFP $^{\text {KIT }}$ and ELFPPURE (applied at the same dilution of $10^{-3}$ ) on saturation of 4MUP hydrolysis in the sample taken from the DYFAMED station on 4 October 2004

in the sample examined, determined in a preceding experiment; Table 1). Final ELFP dilutions ranged from $3 \times 10^{-6}$ to $3 \times 10^{-2}$ (Fig. 1a). To determine $\mathrm{IC}_{50}$, which corresponds to the ELFP concentration (dilution) that causes $50 \%$ inhibition of 4MUP hydrolysis, we expressed the 4MUP hydrolysis velocities occurring in the presence of different ELFP concentrations (dilutions) as percentages of the control values occurring in the absence of ELFP, and plotted them in a semi-logarithmic way as a function of ELFP dilution (Fig. 1a). We calculated $\mathrm{IC}_{50}$ using non-linear regression by fitting data with a decelerating three-parameter sigmoid function (displacement curve), assuming competitive inhibition

$$
v=\operatorname{Min}+(\operatorname{Max}-\operatorname{Min})\left(1+I / I C_{50}\right)^{-1}
$$

where $v$ is the substrate (4MUP) hydrolysis velocity (\% of control), Max and Min are the upper and lower plateaus of 
the displacement curve ( $\%$ of control), respectively, and $I$ is inhibitor concentration (i.e. final ELFP dilution, dimensionless). The lower plateau (Min) of the displacement curve was set to zero, because we usually had not enough data in the range of high ELFP concentrations with which to estimate it reliably (cf. Fig. 1a). Because $\mathrm{IC}_{50}$ values do not directly correspond to $K_{\mathrm{i}}$ unless the substrate concentration used is substantially lower than $K_{\mathrm{M}}$ for this substrate (i.e. $S<0.1 K_{\mathrm{M}}$ : a condition not fulfilled in our experiments), we corrected the $\mathrm{IC}_{50}$ values according to Chang and Prusoff (1973) to obtain inhibition constants $K_{\mathrm{i}}$

$$
K_{\mathrm{i}}=I C_{50}\left(1+S / K_{\mathrm{M}}\right)^{-1}
$$

where $S$ is the substrate (4MUP) concentration used in the displacement experiment, and $K_{\mathrm{M}}$ is the Michaelis constant for 4MUP hydrolysis determined in parallel saturation experiments.

\section{Saturation experiments}

In inhibition experiments of the saturation type, we studied the effect of ELFP on the kinetic parameters $K_{\mathrm{M}}$ and $V_{\max }$ of 4MUP hydrolysis. We measured 4MUP hydrolysis using a series of 4MUP concentrations in the absence (control) and in the presence of a single concentration of ELFP. In each saturation experiment, we tried to choose an inhibitor concentration, $I$, slightly above $K_{\mathrm{i}}$ of ELFP inhibition of 4MUP hydrolysis (determined in parallel inhibition experiments), preferably $K_{\mathrm{i}}<I<2 K_{\mathrm{i}}$. We determined the parameters $V_{\max }$ (maximum hydrolysis velocity) and $K_{\mathrm{M}}$ (Michaelis constant, which reflects enzyme affinity for 4MUP) using non-linear regression by fitting the data with the Michaelis-Menten equation

$$
v=V_{\max } \times S\left(S+K_{\mathrm{M}}\right)^{-1}
$$

where $v$ is 4MUP hydrolysis velocity $\left(\mu \mathrm{mol} \mathrm{L}^{-1} \mathrm{~h}^{-1}\right)$ and $S$ is 4MUP concentration. To obtain $K_{\mathrm{i}}$, the inhibition constant of 4MUP hydrolysis inhibition by ELFP, we used the equation assuming competitive inhibition

$$
K_{\mathrm{i}}=I\left(1+K_{\mathrm{M}}^{\mathrm{CONTROL}} / K_{\mathrm{M}}^{\mathrm{ELFP}}\right)^{-1}
$$

where $K_{\mathrm{M}}^{\mathrm{CONTROL}}$ is the Michaelis constant determined in the absence of the inhibitor, and $K_{\mathrm{M}}^{\mathrm{ELFP}}$ is that determined in the presence of ELFP. The inhibition constants $K_{\mathrm{i}}$ in Eqns 2 and 4 are identical. Consequently, their estimates from displacement and saturation experiments in Eqns 2 and 4, respectively, should theoretically give the same value. The constant $K_{\mathrm{i}}$ numerically corresponds to the concentration of inhibitor necessary for $50 \%$ saturation of enzymes in the absence of substrate, and can be thus interpreted as a measure of enzyme affinity towards the inhibitor, similarly to $K_{\mathrm{M}}$ in the case of a substrate.

\section{Statistical treatment}

The Prism 4 (GraphPad Software, San Diego, USA) statistical package was used to perform non-linear regressions and to test differences between parameter estimates obtained in experiments with inhibitors or control (using the $F$-test).

\section{Results}

The basic hydrological variables and kinetic properties of extracellular phosphatases differed considerably among the samples examined in the present study (Table 1). The soluble reactive phosphorus concentrations varied from the values below detection limit to $220 \mathrm{nmol} \mathrm{L}^{-1}$, $\mathrm{pH}$ ranged from 4.5 to 8.0 , and temperature ranged from 0 to $21^{\circ} \mathrm{C}$. The maximum velocity of $4 \mathrm{MUP}$ hydrolysis, $V_{\max }$, varied within nearly three orders of magnitude $\left(2.6-1600 \mathrm{nmol} \mathrm{L}^{-1} \mathrm{~h}^{-1}\right)$, and the Michaelis constant, $K_{\mathrm{M}}$, varied by two orders of magnitude $\left(0.076-6.3 \mu \mathrm{mol} \mathrm{L}^{-1}\right)$. The lowest values of both parameters were found in marine samples, whereas the highest values occurred in a eutrophic reservoir (Table 1).

In displacement experiments, measured data on inhibition of 4MUP hydrolysis (Fig. 1a) fitted well the competitive inhibition model (Eqn 1). However, in some experiments, the lower plateaus of displacement curves were not sufficiently completed with measured data because of low phosphatase affinity for ELFP (i.e. high $\mathrm{IC}_{50}$, the ELFP concentration causing 50\% inhibition). Theoretically, the inhibitor concentration has to surpass $\mathrm{IC}_{50}$ by at least two orders of magnitude in order to lead to complete inhibition (>99\%; cf. Eqn 1). In contrast, the highest ELFP concentration applied in the present study was substantially (8- to 124-times) higher than $\mathrm{IC}_{50}$ in only 7 of our 12 experiments. In these experiments, the maximum inhibition ranged from 80 to $100 \%$ (average $92 \%$ ), which was not statistically different from the expected 88 to $99 \%$ inhibition (average $93 \%$ ) calculated from Eqn 1 (paired $t$-test, $P=0.73$ ). In the 5 remaining displacement experiments, we observed inhibition only up to $54-81 \%$ owing to the small difference (1.1- to 5.5-fold) between the highest ELFP concentration applied and $\mathrm{IC}_{50}$.

In saturation experiments, the presence of ELFP significantly decreased the apparent $K_{\mathrm{M}}$ of 4MUP hydrolysis compared with the control without ELFP in all cases $(F$-test; $P<0.05)$, while the effect on $V_{\max }$ was statistically significant in 2 of 6 cases only (increase to 120 and $340 \%$ of control value) $(F$-test; $P<0.05)$ The competitive inhibition model expects no effect on $V_{\max }$ and an inhibitor-concentration-dependent (cf. Eqn 4) decrease in $K_{\mathrm{M}}$.

We found the highest affinity of extracellular phosphatases for ELFP (i.e. the lowest $K_{\mathrm{i}}^{\mathrm{PURE}}$; Table 1) in marine samples $\left(0.18-4.5 \mu \mathrm{mol} \mathrm{L}^{-1}\right)$, followed by acidified lakes $(5.2-$ $\left.11 \mu \mathrm{mol} \mathrm{L}^{-1}\right)$ and the freshwater reservoir $\left(16-34 \mu \mathrm{mol} \mathrm{L}^{-1}\right)$. The $K_{\mathrm{M}}$ values of 4MUP hydrolysis in the aquatic systems studies followed the same order as the corresponding $K_{\mathrm{i}}$ values, the latter being on average 8 times higher (excluding the bacterial culture with a difference of two orders of magnitude; Table 1).

Both ELFP ${ }^{\mathrm{PURE}}$ and ELFP ${ }^{\mathrm{KIT}}$ inhibited 4MUP hydrolysis, typically with similar efficiency (see Fig. 1 for examples of raw data). In order to compare inhibition efficiencies of ELFP PURE and ELFP ${ }^{\mathrm{KIT}}$ quantitatively, we determined their respective inhibition constants, $K_{\mathrm{i}}^{\mathrm{PURE}}$ and $K_{\mathrm{i}}^{\mathrm{KIT}}$ (using Eqn 2 or Eqn 4), and calculated ratios of these constants $\left(K_{\mathrm{i}}\right.$-ratio $\left.=K_{\mathrm{i}}^{\mathrm{KIT}} / K_{\mathrm{i}}^{\mathrm{PURE}}\right)$, for each experiment. Fig. 2 shows comparisons of $K_{\mathrm{i}}^{\mathrm{PURE}}$ and $K_{\mathrm{i}}^{\mathrm{KIT}}$ values estimated in displacement (Fig. $2 a$ ) and saturation (Fig. 2b) experiments. Differences between $K_{\mathrm{i}}^{\mathrm{PURE}}$ and $K_{\mathrm{i}}^{\mathrm{KIT}}$ were statistically significant $(P<0.05, F$-test $)$ in 3 of the total of 12 displacement experiments and in 1 of 7 saturation experiments performed: ELFP ${ }^{\mathrm{PURE}}$ was more effective in 1 case 
(a)

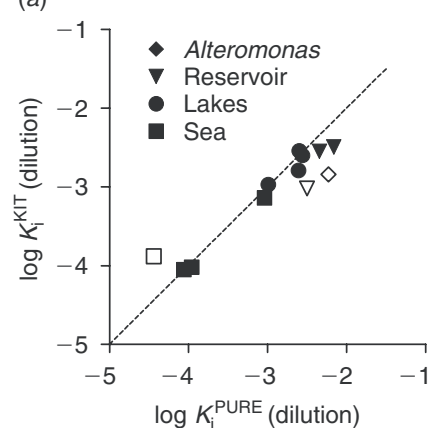

Fig. 2. A comparison of the values of inhibition constants $K_{\mathrm{i}}^{\mathrm{KIT}}$ and $K_{\mathrm{i}}^{\mathrm{PURE}}$, obtained in the experiments in which ELF97 phosphate (purchased from the manufacturer [Molecular Probes] either as a part of the endogenous phosphatase detection kit or as a pure substance) inhibited hydrolysis of 4-methylumbelliferyl phosphate (4MUP) in different aquatic environments (indicated by different symbols). $K_{\mathrm{i}}$ is a measure of enzyme affinity to inhibitor and corresponds to the inhibitor concentration necessary for $50 \%$ saturation of the enzymes. Open symbols indicate cases where $K_{\mathrm{i}}^{\mathrm{KIT}}$ and $K_{\mathrm{i}}^{\mathrm{PURE}}$ differed significantly $(F$-test; $P<0.05)$. Inhibition constants were estimated using two kinds of inhibition experiments: $(a)$ displacement experiments (see Fig. $1 a$ for experimental design) and $(b)$ saturation experiments (cf. Fig. 1b)

$\left(K_{\mathrm{i}}\right.$-ratio $\left.=3.6\right)$ and ELFP ${ }^{\mathrm{KIT}}$ in 3 cases $\left(K_{\mathrm{i}}\right.$-ratios $\left.=0.20-0.31\right)$. The geometrical mean of $K_{\mathrm{i}}$-ratio estimates pooled from displacement and saturation experiments $(n=19)$ was $0.70(95 \%$ confidence interval 0.51-0.95; range 0.20-3.63; median 0.73 ).

Assuming that the differences in efficiencies between ELFP $^{\text {PURE }}$ and ELFP ${ }^{K I T}$ observed in our experiments resulted solely from their different concentrations in the original solutions, we can estimate the original ELFPKIT concentration by dividing the original ELFPPURE ${ }^{\mathrm{PUncentration}}\left(5 \mathrm{mmol} \mathrm{L}^{-1}\right)$ by the mean $K_{\mathrm{i}}$-ratio obtained in our experiments $(0.70)$. This yields an original ELFP ${ }^{\mathrm{KIT}}$ concentration of $7.1 \mathrm{mmol} \mathrm{L}^{-1}$ (95\% confidence interval $5.3-9.8 \mathrm{mmol} \mathrm{L}^{-1}$; calculated from the $95 \%$ confidence limits of $K_{\mathrm{i}}$-ratio as above).

\section{Discussion}

The inhibition approach employed in the present study proved to be a useful tool by which to gain information about the affinity of extracellular phosphatases for ELFP in natural aquatic systems. Direct quantification of ELFP hydrolysis is complicated by low specific fluorescence of the product of ELFP hydrolysis, ELF alcohol (ELFA), and by the complicated time course of ELFA-fluorescence development (Huang et al. 1992; Nedoma et al. 2003b; Dignum et al. 2004). When applying ELFP as an inhibitor of 4MUP hydrolysis, problems imposed by both low ELFA fluorescence and post-hydrolysis ELFA conversions (Huang et al. 1992) are surmounted: enzyme activities as low as $10^{-10} \mathrm{~mol} \mathrm{~L}^{-1} \mathrm{~h}^{-1}$ are measurable using 4MUP, and any processes following the ELFP hydrolysis do not influence results. In the present study, we successfully applied the inhibition approach in environments covering a broad range of phosphatase activities from 2.6 to $1600 \mathrm{nmol} \mathrm{L}^{-1} \mathrm{~h}^{-1}$.

The fact that ELFP interacts with 4MUP hydrolysis does not strictly mean that it is hydrolysed as well; however, we can

reasonably assume that ELFP hydrolysis took place in all cases in which we observed inhibition of 4MUP hydrolysis. ELFP hydrolysis has been proved by direct microscopic observation of ELFA precipitates in acidified lakes Plešné (Nedoma et al. $2003 b$ ) and Certovo (Nedoma and Vrba 2006), in the Rímov Reservoir (Štrojsová et al. 2003, 2005), and in the oligotrophic West Mediterranean Sea and in the Alteromonas infernus cultures (F. Van Wambeke, unpub. data).

The indirect estimates of phosphatase affinity for ELFP in the acidified lakes Plešné $\left(K_{\mathrm{i}}=5.2 \mu \mathrm{mol} \mathrm{L}^{-1}\right)$ and Čertovo $\left(K_{\mathrm{i}}=12-14 \mu \mathrm{mol} \mathrm{L}^{-1}\right)$ obtained in the present study (Table 1$)$ are close to the direct ELFP affinity estimates obtained at Plešné Lake in saturation experiments $\left(K_{\mathrm{M}}=6.5-16.5 \mu \mathrm{mol} \mathrm{L}^{-1}\right.$; Nedoma et al. 2003b). Thus, it seems that $K_{\mathrm{i}}$ represents a good estimation of $K_{\mathrm{M}}$ for ELFP. However, the lack of direct measurements of $K_{\mathrm{M}}$ for ELFP in other systems (with lower phosphatase activity) does not as yet enable us to draw more general conclusions.

The affinity of extracellular phosphatases for ELFP reflected the trophic status of the system examined. The highest affinity $\left(K_{\mathrm{i}} \sim 10^{-7} \mathrm{~mol} \mathrm{~L}^{-1}\right)$ was found at the oligotrophic offshore marine station DYFAMED, the lowest affinity $\left(K_{\mathrm{i}} \sim 10^{-5} \mathrm{~mol} \mathrm{~L}^{-1}\right)$ in the eutrophic freshwater reservoir, with intermediate affinity occurring in oligo- to mesotrophic acidified lakes. It is known that bacterial phosphatases display a higher affinity for 4MUP than do algal phosphatases (Chróst and Overbeck 1987; Vrba et al. 1993; Hoppe 2003); the observed affinity for ELFP might therefore depend on the composition of the microbial community (with respect to its bacterial and phytoplanktonic components) as well. Our data partly support such a relationship: the highest algal biomass and the lowest ELFP affinity occurred in the Rímov Reservoir, while the lowest biomass and the highest affinity were found in marine samples. In contrast, the phosphatase affinity for ELFP in Lake Čertovo was lower than in Plešné Lake (Table 1); however, the former lake is dominated by heterotrophic microorganisms and the latter by algae (Vrba et al. 2003).

The properties of extracellular phosphatases of the cultivated marine bacterium $A$. infernus differed significantly from phosphatases in marine samples: in the bacterial culture, $K_{\mathrm{M}}$ for 4MUP hydrolysis was higher by one order of magnitude and $K_{\mathrm{i}}$ for ELFP was higher by two orders of magnitude compared with sea water. This is a rather common phenomenon, because cultivated microorganisms are adapted to higher concentrations of substrates and nutrients used in cultivations. Consequently, affinity constants measured on bacterial cultures should be extended to natural conditions with caution.

Generally, extracellular phosphatases displayed higher affinity for 4MUP than for ELFP: $K_{\mathrm{M}}$ values of 4MUP hydrolysis were on average almost one order of magnitude lower than $K_{\mathrm{i}}$ values of ELFP (Table 1). This is in agreement with the only available direct comparison of 4MUP and ELFP affinities, measured in parallel saturation experiments in Plešné Lake, whereby $K_{\mathrm{M}}$ of $4 \mathrm{MUP}$ was three-fold lower than $K_{\mathrm{M}}$ of ELFP (Nedoma et al. 2003b). In the present study, the ratio of $K_{\mathrm{M}}$ to $K_{\mathrm{i}}$ fluctuated in the rather broad range between 2 and 18, without a clear trend with respect to the type of locality. The variability in the $K_{\mathrm{M}} / K_{\mathrm{i}}$ ratio was probably partly caused by an experimental error in $K_{\mathrm{M}}$ and $K_{\mathrm{i}}$ determination (variation coefficients for $K_{\mathrm{M}}$ and 
$K_{\mathrm{i}}$ estimates were $9-89 \%$ and $7-43 \%$ respectively). We can also speculate that another source of variability in the $K_{\mathrm{M}} / K_{\mathrm{i}}$ ratio could be connected with different enzymes present in different samples owing to variable plankton composition: different phosphatases may differ in their ability to select between 4MUP and ELFP. The comparatively low ELFP affinity for phosphatases in natural samples should be taken into consideration during planning and interpretation of experiments with ELFP.

The interaction between ELFP and MUP did not deviate considerably from behaviour expected for competitive inhibition. An increase in apparent $K_{\mathrm{M}}$ and no change in $V_{\max }$ for 4MUP in the presence of ELFP are predicted for this type of inhibition (Dixon and Webb 1964). In our experiments, both apparent $K_{\mathrm{M}}$ and $V_{\max }$ were affected by ELFP; however, while apparent $K_{\mathrm{M}}$ consistently and significantly increased in the presence of ELFP, the effect of ELFP on $V_{\max }$ was only weak, irregular (bidirectional) and mostly statistically insignificant. We therefore interpret the interaction of both substrates as competitive.

Our data indicate that all the enzymes cleaving 4MUP are also accessible to ELFP. If the highest ELFP concentration applied was sufficiently above the $\mathrm{IC}_{50}$ value observed in the given experiment, then 4MUP hydrolysis was inhibited completely or nearly completely in the presence of this ELFP concentration (92\% inhibition on average). The relationship inverse to that observed in the present study - a nearly complete inhibition of ELFP hydrolysis in the presence of a high $\left(1 \mathrm{mmol}^{-1}\right)$ concentration of 4MUP (measured with image cytometry) - was reported for Rímov Reservoir (Štrojsová et al. 2003). Both substrates are therefore most probably hydrolysed by the same set of phosphatases.

The original concentrations of ELFP obtained either as pure substance (ELFP PURE; delivered at $5 \mathrm{mmol} \mathrm{L}^{-1}$ concentration) or as part of the endogenous phosphatase detection kit ('Component A', ELFP ${ }^{\mathrm{KIT}}$; delivered at an unspecified concentration) were similar, if not identical. Both ELFP ${ }^{\mathrm{PURE}}$ and ELFP KIT exerted, at the same dilution, comparable effects on 4MUP hydrolysis (Fig. 1). More detailed data analysis revealed that, with $95 \%$ probability, the concentration of ELFP ${ }^{K I T}$ lies in the interval of 5 to $10 \mathrm{mmol} \mathrm{L}^{-1}$ (concentrations of known components of the detection buffer used in the kit are given by Dignum et al. [2004]; $\mathrm{pH}$ is 7.5, Molecular Probes, pers. comm.). To date, the lack of knowledge of the ELFPKIT concentration has to a certain extent limited the interpretation of data obtained with ELFP, because the majority of investigators use the kit in their experiments. It follows from our experiments that the assay ELFP concentration in the endogenous phosphatase detection kit, taking into account the $1 / 20$ dilution of the substrate by detection buffer as recommended by manufacturer, is $250-500 \mu \mathrm{mol} \mathrm{L}^{-1}$. This concentration is about three orders of magnitude higher than our estimates of $K_{\mathrm{i}}$ values for ELFP in oligotrophic marine environments, and is one to two orders of magnitude higher than the same values for freshwater localities (Table 1). Consequently, the ELFP concentration used in the kit should ensure sufficient saturation of phosphatases in natural aquatic environments, specifically $>99.5 \%$ in marine and $90-99 \%$ in freshwater systems. For marine samples, researchers may perhaps consider lowering the ELFP concentration in order to avoid unspecific effects. At any particular locality, it is possible to determine the $K_{\mathrm{i}}$ value for ELFP by applying the inhibition approach suggested in this study, and to decide on an optimum ELFP dilution to be used in experiments.

\section{Acknowledgements}

This study was supported by the GA AV CR research grants A6017202 and IAA600170602, the French program PROOF-PECHE, and the PAI Barrande project 2005-06-009-1. We thank J. Vrba for useful comments on an earlier version of the manuscript.

\section{References}

Chang, Y. C., and Prusoff, W. H. (1973). Relationship between the inhibition constant $\left(\mathrm{K}_{\mathrm{i}}\right)$ and the concentration of inhibitor which causes 50 percent inhibition $\left(\mathrm{IC}_{50}\right)$ of an enzymatic reaction. Biochemical Pharmacology 22, 3099-3108. doi:10.1016/0006-2952(73)90196-2

Chróst, R. J., and Overbeck, J. (1987). Kinetics of alkaline phosphatase activity and phosphorus availability for phytoplankton and bacterioplankton in Lake Plußsee (North German eutrophic lake). Microbial Ecology 13, 229-248. doi:10.1007/BF02025000

Cox, W. G., and Singer, V. L. (1999). A high-resolution, fluorescence-based method for localization of endogenous alkaline phosphatase activity. The Journal of Histochemistry and Cytochemistry 47, 1443-1456.

Dignum, M., Hoogveld, H. L., Matthijs, H. C. P., Laanbroek, H. J., and Pel, R. (2004). Detecting the phosphate status of phytoplankton by enzymelabelled fluorescence and flow cytometry. FEMS Microbiology Ecology 48, 29-38. doi:10.1016/J.FEMSEC.2003.12.007

Dixon, M., and Webb, E. C. (1964). 'Enzymes.' 2nd edn. (Longmans: London.)

Elser, J. J., Stabler, L. B., and Hassett, R. P. (1995). Nutrient limitation of bacterial-growth and rates of bacterivory in lakes and oceans: a comparative study. Aquatic Microbial Ecology 9, 105-110.

Gage, M. A., and Gorham, E. (1985). Alkaline phosphatase activity and cellular phosphorus as an index of the phosphorus status of phytoplankton in Minnesota lakes. Freshwater Biology 15, 227-233. doi:10.1111/J.13652427.1985.TB00195.X

González-Gil, S., Keafer, B. A., Jovine, R. V. M., Aguilera, A., Lu, S., and Anderson, D. M. (1998). Detection and quantification of alkaline phosphatase in single cells of phosphorus-starved marine phytoplankton. Marine Ecology Progress Series 164, 21-35.

Hoppe, H. G. (1983). Significance of exoenzymatic activities in the ecology of brackish water: measurements by means of methylumbelliferyl substrates. Marine Ecology Progress Series 11, 299-308.

Hoppe, H. G. (2003). Phosphatase activity in the sea. Hydrobiologia 493 , 187-200. doi:10.1023/A:1025453918247

Huang, Z., Terpetschnig, E., You, W., and Haugland, R. P. (1992). 2-(2'-phosphoryloxyphenyl)-4(3H)-quinazolinone derivatives as fluorogenic precipitating substrates of phosphatases. Analytical Biochemistry 207, 32-39. doi:10.1016/0003-2697(92)90495-S

Jansson, M., Olsson, H., and Pettersson, K. (1988). Phosphatases: origin, characteristics and function in lakes. Hydrobiologia 170, 157-175.

Jean, N., Boge, G., Jamet, J. L., Richard, S., and Jamet, D. (2003). Seasonal changes in zooplanktonic alkaline phosphatase activity in Toulon Bay (France): the role of Cypris larvae. Marine Pollution Bulletin 46, 346352. doi:10.1016/S0025-326X(02)00450-2

Kopáček, J., Hejzlar, J., Borovec, J., Porcal, P., and Kotorová, I. (2000). Phosphorus inactivation by aluminium in the water column and sediments: a process lowering in-lake phosphorus availability in acidified watershed-lake ecosystems. Limnology and Oceanography 45, 212-225.

Marty, J. C., Chiavérini, J., Pizay, M. D., and Avril, B. (2002). Seasonal and interannual dynamics of nutrients and phytoplankton pigments in the western Mediterranean Sea at the DYFAMED time series station (19911999). Deep-Sea Research. Part II, Topical Studies in Oceanography 49 1965-1985. doi:10.1016/S0967-0645(02)00022-X 
Moutin, T., Thingstad, T. F., Van Wambeke, F., Marie, D., Slawyk, G., Raimbault, P., and Claustre, H. (2002). Does competition for nanomolar phosphate supply explain the predominance of the cyanobacterium Synechococcus? Limnology and Oceanography 47, 1562-1567.

Nedoma, J., and Vrba, J. (2006). Specific activity of cell-surface acid phosphatase in different bacterioplankton morphotypes in an acidified mountain lake. Environmental Microbiology 8, 1271-1279. doi:10.1111/J.1462-2920.2006.01023.X

Nedoma, J., Padisák, J., and Koschel, R. (2003a). Utilisation of 32Plabelled nucleotide- and non-nucleotide dissolved organic phosphorus by freshwater plankton. Archiv fuer Hydrobiologie 58, 87-99.

Nedoma, J., Štrojsová, A., Vrba, J., Komárková, J., and Šimek, K. $(2003 b)$. Extracellular phosphatase activity of natural plankton studied with ELF97 phosphate: fluorescence quantification and labelling kinetics. Environmental Microbiology 5, 462-472. doi:10.1046/J.14622920.2003.00431.X

Rengefors, K., Petterson, K., Blenckner, T., and Anderson, D. M. (2001). Species-specific alkaline phosphatase activity in freshwater spring phytoplankton: application of a novel method. Journal of Plankton Research 23, 435-443. doi:10.1093/PLANKT/23.4.435

Schindler, D. W. (1977). Evolution of phosphorus limitation in lakes. Science 195, 260-262. doi:10.1126/SCIENCE.195.4275.260

Štrojsová, A., Vrba, J., Nedoma, J., Komárková, J., and Znachor, P. (2003). Seasonal study of extracellular phosphatase expression in the phytoplankton of a eutrophic reservoir. European Journal of Phycology 38, 295-306. doi:10.1080/09670260310001612628
Štrojsová, A., Vrba, J., Nedoma, J., and Šimek, K. (2005). Extracellular phosphatase activity of freshwater phytoplankton exposed to different in situ phosphorus concentrations. Marine and Freshwater Research 56, 417-424. doi:10.1071/MF04283

Taylor, W. D., and Lean, D. R. S. (1991). Phosphorus pool sizes and fluxes in the epilimnion of a mesotrophic lake. Canadian Journal of Fisheries and Aquatic Sciences 48, 1293-1301.

Van Wambeke, F., Christaki, U., Giannakourou, A., Moutin, T., and Souvemerzoglou, K. (2002). Longitudinal and vertical trends of bacterial limitation by phosphorus and carbon in the Mediterranean Sea. Microbial Ecology 43, 119-133. doi:10.1007/S00248-001-0038-4

Vrba, J., Komárková, J., and Vyhnálek, V. (1993). Enhanced activity of alkaline phosphatases - phytoplankton response to epilimnetic phosphorus depletion. Water Science and Technology 28, 15-24.

Vrba, J., Vyhnálek, V., Hejzlar, J., and Nedoma, J. (1995). Comparison of phosphorus deficiency indices during a spring phytoplankton bloom in a eutrophic reservoir. Freshwater Biology 33, 73-81. doi:10.1111/J.13652427.1995.TB00387.X

Vrba, J., Kopáček, J., Fott, J., Kohout, L., Nedbalová, L., Pražáková, M., Soldán, T., and Schaumburg, J. (2003). Long-term studies (18712000) on acidification and recovery of lakes in the Bohemian Forest (central Europe). Science of the Total Environment 310, 73-85. doi:10.1016/S0048-9697(02)00624-1

Manuscript received 7 November 2006, accepted 28 February 2007 